Revista Brasileira de Meteorologia, v.26, n.2, 215 - 224, 2011

\title{
ESTIMATIVA DA RADIAÇÃO DE ONDA LONGA ATMOSFÉRICA EM ÁREAS DE FLORESTA E DE PASTAGEM NO SUDOESTE DA AMAZÔNIA
}

\author{
LEONARDO JOSÉ GONÇALVES AGUIAR ${ }^{1}$, JOSÉ MARIA NOGUEIRA DA COSTA ${ }^{1}$, GRACIELA \\ REDIES FISCHER ${ }^{1}$, RENATA GONÇALVES AGUIAR ${ }^{2}$, ANTÔNIO CARLOS LÔLA DA COSTA ${ }^{3}$, \\ WILLIAMS PINTO MARQUES FERREIRA ${ }^{4}$
}

\author{
${ }^{1}$ Universidade Federal de Viçosa (UFV), Departamento de Engenharia Agrícola, Viçosa, MG, Brasil. \\ ${ }^{2}$ Fundação Universidade Federal de Rondônia (UNIR), Departamento de Engenharia Ambiental, Ji-Paraná, \\ RO, Brasil. \\ ${ }^{3}$ Universidade Federal do Pará (UFPA), Departamento de Meteorologia, Belém, PA, Brasil. \\ ${ }^{4}$ Empresa Brasileira de Pesquisa Agropecuária (EMBRAPA), EMBRAPA Milho e Sorgo, Sete Lagoas, MG, \\ Brasil.
}

veraneiro@yahoo.com.br,jmncosta@yahoo.com, gracielafischer@gmail.com,rgaguiar@gmail.com, lola@ufpa.br,williams@cnpms.embrapa.br

Recebido Maio 2010 - Aceito Novembro 2010

\section{RESUMO}

A radiação de onda longa proveniente da atmosfera $\left(\mathrm{L}_{\text {in }}\right)$ é a componente do balanço de radiação mais difícil de ser medida. Na Amazônia praticamente não existem medidas regulares dessa componente, mesmo sendo uma importante variável no cálculo do balanço de radiação à superfície e muito usada para alimentar modelos climáticos. Tendo em vista a necessidade desses dados, o objetivo do presente trabalho é avaliar o desempenho de sete equações na estimativa da $\mathrm{L}_{\text {in }}$ para dias de céu claro em áreas de floresta (Reserva Biológica do Jaru, 10 4'48"S; 61 $55^{\circ} 48^{\prime \prime} \mathrm{W}$ ) e de pastagem (Fazenda Nossa Senhora, $\left.10^{\circ} 45^{\prime} \mathrm{S} ; 62^{\circ} 21^{\prime} \mathrm{W}\right)$ no sudoeste da Amazônia. Medidas de radiação de onda longa atmosférica realizadas no período de junho de 2005 a maio de 2006 foram comparadas com as estimativas. As equações testadas tiveram desempenho satisfatório apenas durante a estação seca. As condições de alta nebulosidade, dominantes na estação chuvosa, restringiram a quantidade de dados utilizados na avaliação das equações. As equações que utilizam informações de temperatura do ar e pressão de vapor d'água para a estimativa da $\mathrm{L}_{\text {in }}$ tiveram melhor desempenho em relação às que utilizam apenas a temperatura do ar. As equações de Brutsaert (1975), Idso (1981) e Prata (1996) foram as que apresentaram melhor desempenho, apresentando os maiores índices de concordância, $\mathrm{e}$ sendo, portanto, as equações mais indicadas para a estimativa da radiação de onda longa atmosférica no sudoeste da Amazônia.

Palavras-Chave: Condição de céu claro, temperatura do ar, pressão de vapor d'água.

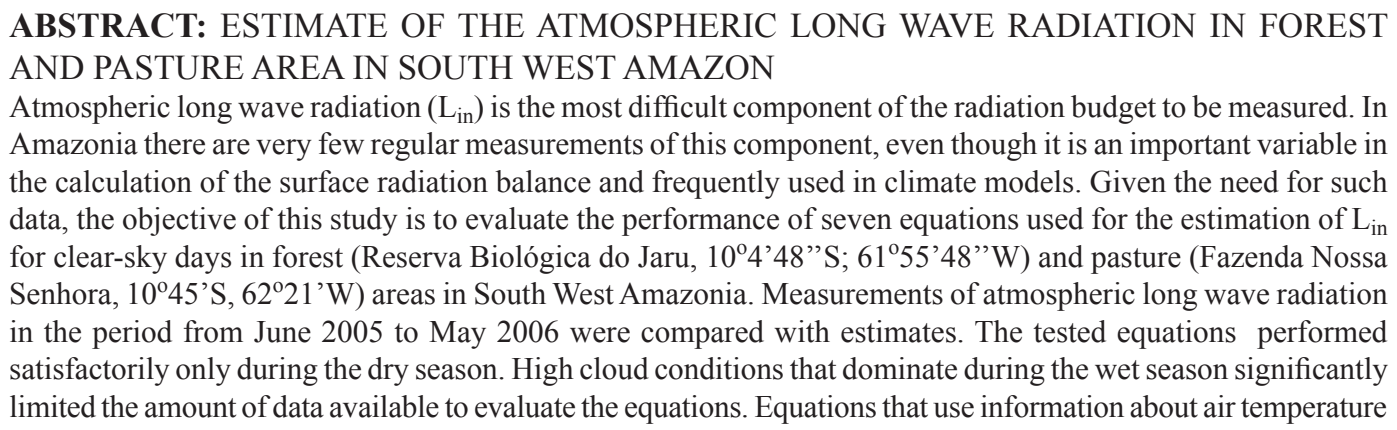


and vapor pressure for the $\mathrm{L}_{\mathrm{in}}$ estimate performed better than those that use only air temperature. The equations of Brutsaert (1975), Idso (1981) and Prata (1996) performed best, had the highest rates of agreement and are therefore the most appropriate equations for estimating atmospheric long wave radiation in South West Amazonia.

Keywords: Clear-Sky condition, air temperature, water vapor pressure.

\section{INTRODUÇÃO}

A radiação de onda longa, compreendida entre 4 e 100 $\mu \mathrm{m}$, é o fluxo radiante de energia resultante da emissão dos gases atmosféricos e de superfícies liquidas e sólidas da Terra (Galvão e Fisch, 2000). A radiação de onda longa proveniente da atmosfera é a componente do balanço de radiação mais difícil de ser medida. Embora haja instrumentos para sua medida, esses emitem radiação em comprimentos de onda e intensidade comparáveis aos da suposta medida (Von Randow e Alvalá, 2006), necessitando assim, de correções realizadas a partir da temperatura desses instrumentos.

$\mathrm{Na}$ Região Amazônica praticamente não existem medidas regulares de radiação de onda longa da atmosfera, mesmo sendo essa uma variável importante no cálculo do balanço de radiação à superfície, pois representa a contribuição da atmosfera e engloba informações de nebulosidade e concentração de vapor d'água (Galvão e Fisch, 2000). A maioria dos resultados publicados do balanço de radiação de ondas longas é estimada ou obtida como resíduo a partir da equação do balanço de radiação, tais como os estudos realizados por Manzi et al. (1986), André et al. (1988), Bastable et al. (1993), Ribeiro (1994), Culf et al. (1996), Feitosa (1996) e Reschke (1996), na floresta Amazônica.

Outra alternativa para se obter esses dados é através de equações empíricas e analíticas que estimam a radiação de onda longa atmosférica a partir de valores de temperatura do ar e da pressão de vapor d'água medidos ao nível do abrigo meteorológico (Prata, 1996). Uma inconveniência é que a maioria dessas equações apenas estima a radiação de onda longa atmosférica para dias de céu claro. Além disso, estimativas horárias, frequentemente exigidas em modelos de balanço de energia à superfície, são mais sujeitas a erros, apresentando melhor desempenho quando se consideram bases diárias ou médias de longo prazo.

Com o intuito de avaliar o desempenho de equações que estimam a radiação de onda longa atmosférica, alguns estudos têm sido desenvolvidos, como os de Galvão e Fisch (2000) e Correia (2000), para a Região Amazônia e Von Randow e Alvalá (2006) para o Pantanal Sul Mato-Grossense. Entretanto, esses estudos apenas avaliaram as equações para a estação seca ou final da estação chuvosa e início da seca, não avaliando para o período chuvoso.

Tendo em vista a necessidade de se obter valores de radiação de onda longa atmosférica, tanto no período seco quanto no chuvoso, este trabalho tem a finalidade de avaliar o desempenho de equações que estimam a radiação de onda longa atmosférica em áreas de floresta e pastagem no sudoeste da Amazônia no período de junho de 2005 a maio de 2006.

\section{MATERIAL E MÉTODOS}

\subsection{Descrição das áreas de estudo}

O presente estudo foi realizado em dois sítios experimentais pertencentes à rede de torres do Programa de Grande Escala da Biosfera-Atmosfera na Amazônia - LBA, localizados no Estado de Rondônia, sendo um sítio de pastagem e outro de floresta.

O sítio de pastagem está situado na Fazenda Nossa Senhora (FNS) $\left(10^{\circ} 45^{\prime} \mathrm{S} ; 62^{\circ} 21^{\prime} \mathrm{W}, 290 \mathrm{~m}\right)$, próximo a Ouro Preto d'Oeste, e encontra-se no centro de uma área desmatada com aproximadamente $50 \mathrm{~km}$ de raio. Tem como cobertura vegetal predominante a gramínea Brachiaria brizantha.

O sítio de floresta localiza-se na Reserva Biológica do Jaru (Rebio Jaru) (104'48”S; 61 ${ }^{\circ} 55^{\prime} 48^{\prime}$ 'W, 120 m), situada no município de Ji-Paraná, possuindo uma área de 330.000 hectares, classificada como Floresta Ombrofila Aberta (Culf et al., 1997). Sua vegetação é característica de terra firme, com altura média do dossel de aproximadamente $35 \mathrm{~m}$, sendo que algumas árvores emergentes podem atingir até $45 \mathrm{~m}$.

\subsection{Aquisição dos dados}

Para o desenvolvimento deste trabalho foram realizadas medições contínuas de: irradiância solar global $\left(\mathrm{S}_{\text {in }}\right)$, através de um piranômetro CM21 (Kipp\&Zonen); radiação de onda longa atmosférica $\left(\mathrm{L}_{\text {in }}\right)$, por um pirgeômetro CG1 (Kipp\&Zonen); precipitação (P), por um pluviômetro EM ARG-100 (Environmental Measurements LTD); temperatura (T) e umidade relativa do ar (UR), usando um termohigrômetro HMP45D (Vaisala Inc.). Esses dados foram coletados durante 12 meses, no período de junho de 2005 a maio de 2006, em duas torres micrometeorológicas, uma com $10 \mathrm{~m}$ de altura, no sítio de pastagem, e outra com $61,5 \mathrm{~m}$ de altura, no de floresta. As leituras foram realizadas em intervalos de 30 segundos, e médias armazenadas a cada 10 minutos em um datalogger CR10X na pastagem e por um datalogger CR23X na floresta, ambos fabricados pela Campbell Scientific Instrument, Utah, USA. Os sensores de radiação estavam instalados a uma altura de $6,5 \mathrm{~m}$ na pastagem e $58 \mathrm{~m}$ na floresta. Já os de temperatura e umidade 
relativa do ar se encontravam a 8,3 e $61 \mathrm{~m}$, respectivamente. Os pluviômetros estavam a $0.5 \mathrm{~m}$ na pastagem e $61 \mathrm{~m}$ na floresta.

Os cálculos de radiação de onda longa atmosférica foram realizados a partir das equações de Brunt (1932), Swinbank (1963), Idso e Jackson (1969), Brutsaert (1975), Satterlund (1979), Idso (1981) e Prata (1996) (Tabela 1).

A pressão de saturação do vapor d'água ( $\left.e_{s}, \mathrm{em} \mathrm{hPa}\right)$, foi calculada a partir da equação de Tetens (1930) para temperatura maior ou igual a $0{ }^{\circ} \mathrm{C}$ (Equação 1). A pressão atual do vapor d'água (e) foi calculada utilizando a $e_{s}$ e a umidade relativa (UR) (Equação 2).

$$
\begin{aligned}
& e_{s}=6,1078 \times 10^{\frac{7,5 t}{(237,3+t)}} \\
& e=\frac{U R e_{s}}{100}
\end{aligned}
$$

Como as equações utilizadas no cálculo da radiação de onda longa atmosférica foram desenvolvidas para estimá-la apenas sob condições de céu claro, e por não haver informações de nebulosidade, foi feita a caracterização de dias de céu claro ou com poucas nuvens com base na razão entre a irradiância solar global e a irradiância extraterrestre $\left(\mathrm{S}_{\text {in }} / \mathrm{R}_{\mathrm{o}}\right)$, utilizando a mesma metodologia empregada por Culf et al. (1995), Galvão e Fisch (2000) e Von Randow e Alvalá (2006). Os dias considerados como de céu claro ou com poucas nuvens, foram aqueles em que a razão entre a $S_{\text {in }}$ e a $R_{0}$ foi superior a 0,5 . A radiação solar extraterrestre diária e horária foi estimada usando a relação geométrica Terra-Sol (Iqbal, 1983) e a constante solar $1367 \mathrm{~W} \mathrm{~m}^{-2}$.

\subsection{Análise estatística}

O desempenho das equações utilizadas para estimar a radiação de onda longa atmosférica foi avaliado estatisticamente através da raiz do erro médio quadrático (RMSE, em inglês) (Equação 3), do erro de viés médio (MBE, em inglês) (Equação
4), do coeficiente de determinação $\left(\mathrm{r}^{2}\right)$ (Equação 5) e do índice de concordância de Willmott (d) (Equação 6). Esses testes estatísticos foram utilizados para descrever as correlações entre os valores estimados pelas equações e os valores medidos.

$$
\begin{aligned}
& \text { RMSE }=\left(\frac{1}{N} \sum_{i=1}^{N}\left(y_{i}-x_{i}\right)^{2}\right)^{1 / 2} \\
& M B E=\frac{1}{N} \sum_{i=1}^{N}\left(y_{i}-x_{i}\right) \\
& r^{2}=\left[\frac{\left[\sum_{i=1}^{N}\left(y_{i}-\bar{y}\right)\left(x_{i}-\bar{x}\right)\right]^{2}}{\left[\sum_{i=1}^{N}\left(y_{i}-\bar{y}\right)^{2}\right]\left[\sum_{i=1}^{N}\left(x_{i}-\bar{x}\right)^{2}\right]}\right]
\end{aligned}
$$

em que, $N$ é o número de dados da amostra, $y$ refere-se aos valores estimados, $x$ aos valores medidos e a barra acima da variável corresponde ao valor médio da variável.

\section{RESULTADOS E DISCUSSÃO}

\subsection{Análise das variações sazonais da temperatura do ar e do déficit da pressão de vapor d'água}

Antes de avaliar as equações de estimativa da $\mathrm{L}_{\text {in }}$, faz-se necessário um estudo das variações sazonais da temperatura do ar e do déficit da pressão de vapor d'água, visto que as condições da atmosfera, bem como seus constituintes, principalmente o vapor d'água, influenciam fortemente na quantidade de radiação de onda longa atmosférica que é emitida para a superfície da Terra.

A temperatura média mensal do ar apresentou pequena variação sazonal, cerca de $3{ }^{\circ} \mathrm{C}$, tanto no sítio de floresta, de 23 a $26^{\circ} \mathrm{C}$, quanto no de pastagem, de 24 a $27^{\circ} \mathrm{C}$ (Figura 1). A temperatura média mensal máxima do ar variou entre $32 \mathrm{e}$ $36{ }^{\circ} \mathrm{C}$ na floresta e entre 32 e $35^{\circ} \mathrm{C}$ na pastagem, com valores maiores encontrados nos meses de setembro na floresta, 36 ${ }^{\circ} \mathrm{C}$, e de agosto na pastagem, $35{ }^{\circ} \mathrm{C}$. Os menores valores da

Tabela 1 - Equações utilizadas para estimar a radiação de onda longa atmosférica, em que $\sigma$ é a constante de Stefan-Boltzmann $\left(5,6697 x 10^{-8} \mathrm{~W}\right.$ $\left.\mathrm{m}^{-2} \mathrm{~K}^{-4}\right)$, e a pressão de vapor d'água ( $\left.\mathrm{hPa}\right), \mathrm{T}$ a temperatura do $\operatorname{ar}(\mathrm{K})$ e $\xi=46,5(\mathrm{e} / \mathrm{T})$.

\begin{tabular}{ll}
\hline Equações & Parametrização \\
\hline Brunt (1932) & $\mathrm{L}_{\text {in }}=\left[0,065\left(e^{0,5}\right)+0,52\right] \sigma \mathrm{T}^{4}$ \\
Swinbank (1963) & $\mathrm{L}_{\text {in }}=\left[9,2 \times 10^{-6}\right] \sigma \mathrm{T}^{6}$ \\
Idso e Jackson (1969) & $\mathrm{L}_{\text {in }}=\left\{1-0,26 \exp \left[-7,77 \times 10^{-4}(273-\mathrm{T})^{2}\right]\right\} \sigma \mathrm{T}^{4}$ \\
Brutsaert (1975) & $\mathrm{L}_{\text {in }}=\left[1,24(e / \mathrm{T})^{1 / 7}\right] \sigma \mathrm{T}^{4}$ \\
Satterlund (1979) & $\mathrm{L}_{\text {in }}=\left\{1,08\left[1-\exp \left(-e^{\mathrm{T} / 2016}\right)\right]\right\} \sigma \mathrm{T}^{4}$ \\
Idso (1981) & $\mathrm{L}_{\text {in }}=\left[0,7+5,95 \times 10^{-5}(e \exp (1500 / \mathrm{T}))\right] \sigma \mathrm{T}^{4}$ \\
Prata (1996) & $\mathrm{L}_{\text {in }}=\left\{1-(1+\xi) \exp \left[-(1,2+3 \xi)^{0,5}\right]\right\} \sigma \mathrm{T}^{4}$ \\
\hline
\end{tabular}


temperatura média mensal mínima do ar foram encontrados no mês de agosto, em ambos os sítios, com valores de $15^{\circ} \mathrm{C}$ na floresta e $13^{\circ} \mathrm{C}$ na pastagem. A variação da temperatura média mensal mínima do ar foi de cerca de $7{ }^{\circ} \mathrm{C}$ na floresta, entre 15 e $22{ }^{\circ} \mathrm{C}$, e de $8{ }^{\circ} \mathrm{C}$ na pastagem, entre 13 e $21^{\circ} \mathrm{C}$. A ocorrência de temperaturas mínimas mais baixas no período de junho a setembro deve-se tanto à influência de eventos de "friagens", em que massas de ar frio alcançam o norte do Brasil, porém com intensidade reduzida, quanto a uma menor cobertura de nuvens, permitindo que haja maior perda de calor pela superfície durante a noite.

A semelhança no comportamento do ciclo médio da temperatura do ar no sítio de floresta e de pastagem na estação chuvosa (período compreendido entre os meses de janeiro e março), com diferença de aproximadamente $1{ }^{\circ} \mathrm{C}$ durante todo o dia, deve-se à similaridade na cobertura de nuvens entre os dois sítios (Figura 2). Por outro lado, na estação seca (junho a agosto) há uma grande diferença na cobertura de nuvens entre os dois sítios, o que pode ser percebido pelo comportamento do ciclo médio da temperatura do ar, com temperaturas mais baixas no período noturno, indicando maior perda radiativa pela superfície de pastagem, bem como pela maior amplitude térmica, cerca de $12{ }^{\circ} \mathrm{C}$ na pastagem, enquanto na floresta foi de $10{ }^{\circ} \mathrm{C}$. Na estação chuvosa, a amplitude térmica reduziu-se a cerca de metade da observada na estação seca, com valor de $5{ }^{\circ} \mathrm{C}$ em ambos os sítios.

$\mathrm{Na}$ estação chuvosa as curvas do déficit de pressão de vapor d'água $(\Delta \mathrm{e})$ no sítio de floresta são bem próximas às do sítio de pastagem, com maior diferença ocorrendo na estação seca (Figura 3). Conforme esperado, os maiores déficits de pressão de vapor d'água ocorreram na estação seca (junho a agosto), com valores máximos em agosto, de $16,66 \mathrm{hPa}$ na

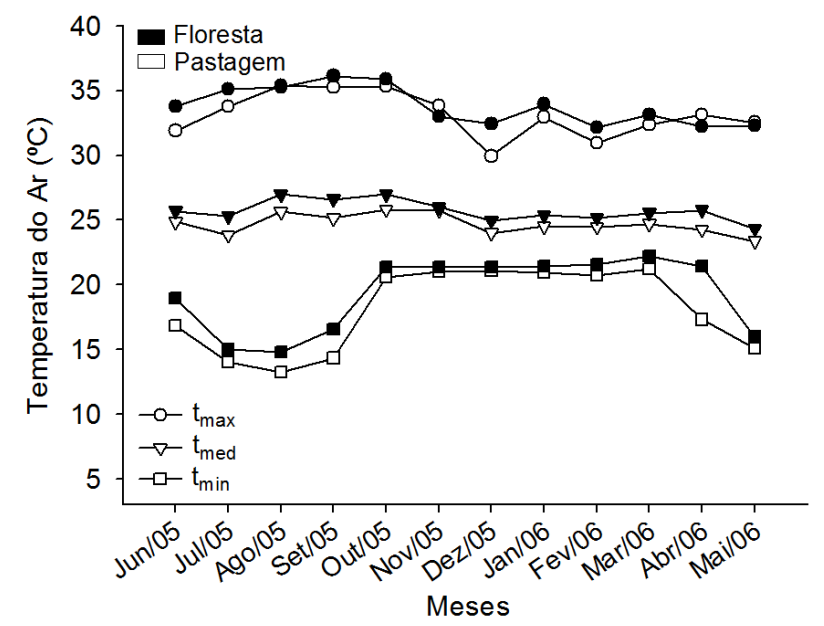

Figura 1 - Valores médios mensais das temperaturas do ar máxima, média e mínima para os sítios de pastagem e de floresta, de junho de 2005 a maio de 2006.

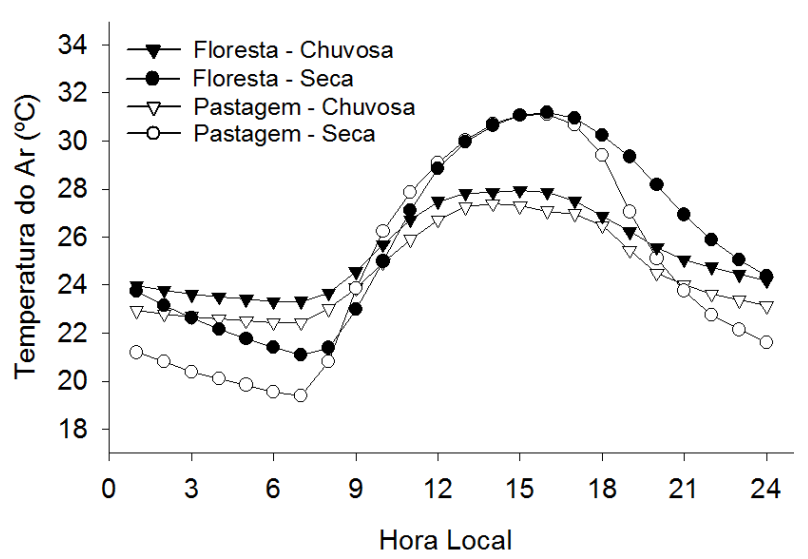

Figura 2 - Ciclo diário médio da temperatura do ar nas estações chuvosa (janeiro a março) e seca (junho a agosto) para os sítios de pastagem e de floresta.

pastagem e 15,31 hPa na floresta (Figura 3). Nos meses mais secos, o déficit de pressão de vapor se manteve menor na região de floresta, o que indica haver uma maior disponibilidade de água para ser evapotranspirada na floresta, visto que as árvores possuem raízes mais profundas, atingindo o lençol freático nas camadas inferiores do solo (Silva Dias et al., 2005). Von Randow et al. (2004) relatam que mesmo após um longo período de seca, a floresta pode manter uma grande retirada de água do solo. Por outro lado, as gramíneas sofrem mais com grandes períodos sem precipitação, uma vez que seu sistema radicular é mais curto.

\subsection{Estimativa da radiação de onda longa atmosférica}

Para avaliar o desempenho das equações propostas por Brunt (1932), Swinbank (1963), Idso e Jackson (1969), Brutsaert (1975), Satterlund (1979), Idso (1981) e Prata (1996), na estimativa da radiação de onda longa atmosférica $\left(\mathrm{L}_{\mathrm{in}}\right)$, foram utilizados valores médios diários de $\mathrm{L}_{\text {in }}$ nos dias caracterizados como de céu claro ou com poucas nuvens, ou seja, os que tiveram valores da razão entre a irradiância solar global e a radiação solar extraterrestre $\left(R_{0}\right)$ superiores a 0,5 . Na Tabela 2 são apresentados o número de dias em que houve medidas e que atenderam à condição de céu claro em cada mês do período estudado.

Após as estimativas, os valores de $\mathrm{L}_{\text {in }}$ foram separados por mês e calculou-se a raiz do erro médio quadrático (RMSE), o erro de viés médio (MBE), o coeficiente de determinação $\left(\mathrm{r}^{2}\right)$ e o índice de concordância de Willmott (d).

Maiores erros nos meses referentes à estação chuvosa (janeiro a março) demonstram que essas equações não se ajustam bem a locais muito úmidos, como é o caso do sudoeste da Amazônia durante o período chuvoso, em que o RMSE, MBE e $\mathrm{r}^{2}$ variaram de 6,66 a $52,81 \mathrm{~W} \mathrm{~m}^{-2},-51,95$ a $2,20 \mathrm{~W} \mathrm{~m}^{-2}$ 


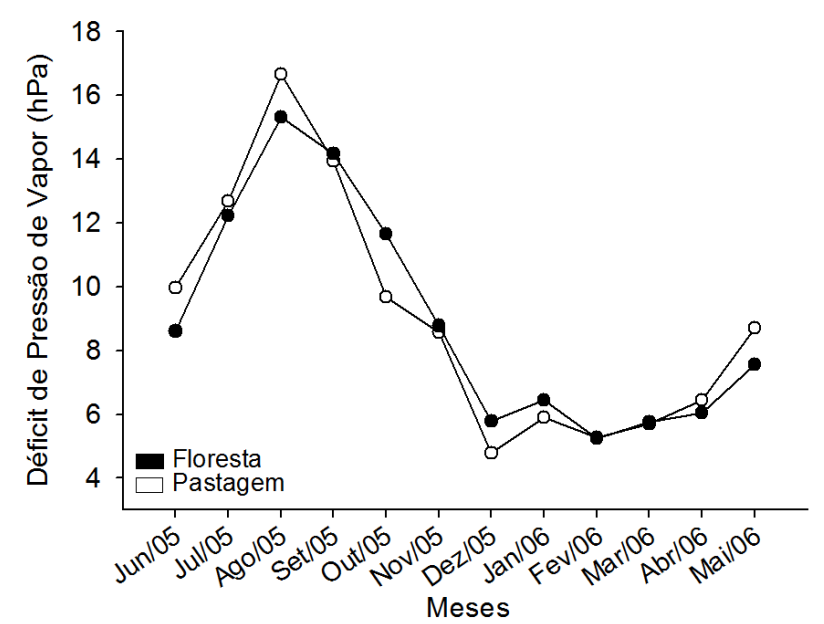

Figura 3 - Valores médios mensais do déficit de pressão de vapor d'água para os sítios de pastagem e de floresta, de junho de 2005 a maio de 2006.

e de 0,00 a 0,29 , no sítio de pastagem (Tabela 3 ), e de 5,06 a $52,16 \mathrm{~W} \mathrm{~m}^{-2},-51,77$ a $4,84 \mathrm{~W} \mathrm{~m}^{-2}$ e de 0,00 a 0,06 , no sítio de floresta (Tabela 4), respectivamente. Por outro lado, nos meses referentes à estação seca (junho a agosto) as equações tiveram desempenho satisfatório, com variação do RMSE, MBE e $\mathrm{r}^{2}$ de 5,49 a $20,32 \mathrm{~W} \mathrm{~m}^{-2},-18,34$ a $19,84 \mathrm{~W} \mathrm{~m}^{-2}$ e de 0,45 a 0,98 , no sítio de pastagem (Tabela 3), e de 6,38 a $29,25 \mathrm{~W} \mathrm{~m}^{-2},-28,94$ a $18,04 \mathrm{~W} \mathrm{~m}^{-2}$ e de 0,50 a 0,93 , no sítio de floresta (Tabela 4), respectivamente.

Através do MBE, em que valores negativos indicam subestimativa e valores positivos superestimativa, nota-se que há uma tendência das equações a subestimar a $\mathrm{L}_{\text {in }}$, exceto pela equação de Idso (1981), que na maioria das vezes superestimou-a. Essa tendência de subestimativa pela maioria das equações também foi encontrada por Von Randow e Alvalá (2006), que indicaram que o problema poderia estar relacionado aos coeficientes utilizados nas equações, os quais foram ajustados para outras regiões.

A equação de Brutsaert (1975) obteve os maiores índices de concordância nos meses referentes às estações chuvosa e seca para o sítio de pastagem, evidenciando menores erros tanto em relação aos dados estimados, quanto aos observados, mesmo não tendo, na maioria das vezes, os melhores coeficientes de determinação, os quais retratam apenas os erros em relação a equação de regressão. Nos meses referentes à estação de transição seca-chuvosa (setembro a dezembro) a equação de melhor desempenho foi a de Idso (1981), seguida por Brutsaert (1975). A equação de Prata (1996) apresentou resultados bem semelhantes aos de Brutsaert (1975), porém, sempre inferiores. Por sua vez, as equações de Swinbank (1963) e de Idso e Jackson (1969), que consideram apenas a temperatura do ar, tiveram, na maioria das vezes, os piores coeficientes de determinação
Tabela 2 - Número de dias utilizados para estimar a $\mathrm{L}_{\text {in }}$ no período de junho de 2005 a maio de 2006 nos sítios de pastagem e floresta.

\begin{tabular}{lcc}
\hline Mês & Pastagem & Floresta \\
\hline Junho & 23 & 8 \\
Julho & 29 & 30 \\
Agosto & 26 & 28 \\
Setembro & 20 & 15 \\
Outubro & 18 & 17 \\
Novembro & 14 & 0 \\
Dezembro & 0 & 3 \\
Janeiro & 5 & 12 \\
Fevereiro & 8 & 6 \\
Março & 9 & 9 \\
Abril & 11 & 8 \\
Maio & 21 & 19 \\
\hline
\end{tabular}

e índices de concordância. No sítio de floresta a equação de Brutsaert (1975) obteve melhor desempenho nos meses de maio a agosto e a de Idso (1981) nos de setembro a abril, considerando como referência o índice de concordância.

Comparando os resultados deste trabalho com os encontrados por Galvão e Fisch (2000), para a área de pastagem, Correia (2000) em uma região de policultivo na Amazônia, e Von Randow e Alvalá (2006) para o Pantanal Sul Mato-Grossense, pode-se destacar que os valores de RMSE e MBE obtidos neste trabalho, para os meses referentes à estação seca, são, na maioria das vezes, menores, indicando maior proximidade entre os valores da $L_{\text {in }}$ estimados e medidos. Isso pode ter ocorrido devido a 2005 ter sido um ano diferenciado, sendo mais seco do que o habitual, e estando, provavelmente, mais próximo das condições atmosféricas às quais os coeficientes das equações foram ajustados. As equações com maiores coeficientes de determinação, encontrados neste trabalho, foram as de Idso (1981) e de Brunt (1932) para o sítio de pastagem e de Idso (1981), Prata (1996) e Brunt (1932) para o de floresta. Nos estudos de Galvão e Fisch (2000) e Von Randow e Alvalá (2006) prevaleceram as equações de Swinbank (1963) e Idso e Jackson (1969). Já para Correia (2000), as equações com maiores coeficientes de determinação foram as de Swinbank (1963) e Satterlund (1979). Deve-se ressaltar que Galvão e Fisch (2000) e Correia (2000) não avaliaram as equações de Idso (1981) e Prata (1996).

Ao analisar os ciclos médios diários da radiação de onda longa atmosférica observada e estimada pelas equações avaliadas, nota-se que as equações tanto subestimam, quanto superestimam a radiação de onda longa atmosférica ao longo do dia, tanto na pastagem (Figura 4) quanto na floresta (Figuras 5).

Nos meses com maior déficit de pressão de vapor d'água (julho a setembro), a equação de Swinbank (1963) é a que mais subestima a radiação de onda longa atmosférica no período 
noturno, concordando com os resultados encontrados por Galvão e Fisch (2000), onde os autores relatam que as equações que levam em consideração apenas a temperatura do ar, foram as que mais subestimaram a radiação de onda longa atmosférica durante o período noturno. Por outro lado, no período diurno as piores estimativas são apresentadas pelas equações de Brunt (1932) e Idso e Jackson (1969).

Nos meses mais úmidos (novembro a abril) a equação de Swinbank (1963) obteve o pior desempenho durante todo o dia. Por sua vez, a equação de Idso (1981) se mostrou mais eficiente nos meses mais úmidos, onde ocorrem menores amplitudes térmicas, enquanto que nos mais secos se percebe maiores erros nas estimativas, principalmente no período noturno, o que demonstra a sensibilidade dessa equação à variação da temperatura do ar, uma vez que a pressão de vapor d'água praticamente não varia ao longo do dia. Resultados semelhantes a esses observados nos meses mais secos também foram encontrados por Von Randow e Alvalá (2006).

\section{CONCLUSÕES}

As equações testadas tiveram desempenho satisfatório apenas durante a estação seca. As condições de alta nebulosidade, dominantes na estação chuvosa, restringiram a quantidade de

Tabela 3 - Estatísticas relacionando os valores da radiação de onda longa atmosférica observados e estimados pelas equações de Brunt (1932) (Equação 1), Swinbank (1963) (Equação 2), Idso e Jackson (1969) (Equação 3), Brutsaert (1975) (Equação 4), Satterlund (1979) (Equação 5), Idso (1981) (Equação 6) e Prata (1996) (Equação 7), para os meses de junho de 2005 a maio de 2006 no sítio de pastagem. A raiz do erro médio quadrático (RMSE) e o erro de viés médio (MBE) estão em W m${ }^{-2}$, o coeficiente de determinação (r2) e o índice de concordância (d) são adimensionais.

\begin{tabular}{lcccccccccccc}
\hline & \multicolumn{4}{c}{ Junho/05 } & \multicolumn{4}{c}{ Julho/05 } & \multicolumn{4}{c}{ Agosto/05 } \\
\hline Equação & RMSE & MBE & $\mathbf{r}^{2}$ & d & RMSE & MBE & $\mathbf{r}^{2}$ & d & RMSE & MBE & $\mathbf{r}^{2}$ & d \\
Eq. 1 & 15,14 & $-14,33$ & 0,82 & 0,57 & 17,45 & $-15,23$ & 0,75 & 0,76 & 17,14 & $-16,35$ & 0,97 & 0,88 \\
Eq. 2 & 19,55 & $-18,34$ & 0,61 & 0,46 & 14,54 & $-6,91$ & 0,45 & 0,76 & 12,28 & $-1,36$ & 0,87 & 0,91 \\
Eq. 3 & 10,75 & $-8,46$ & 0,61 & 0,61 & 13,19 & 2,55 & 0,46 & 0,81 & 14,60 & 8,85 & 0,87 & 0,89 \\
Eq. 4 & 5,49 & $-1,19$ & 0,80 & 0,84 & 8,81 & 0,19 & 0,73 & 0,92 & 6,34 & $-0,83$ & 0,96 & 0,98 \\
Eq. 5 & 6,39 & $-0,17$ & 0,73 & 0,73 & 13,11 & 7,72 & 0,62 & 0,81 & 13,83 & 8,76 & 0,93 & 0,90 \\
Eq. 6 & 20,32 & 19,84 & 0,85 & 0,47 & 18,08 & 16,20 & 0,78 & 0,78 & 14,23 & 13,48 & 0,98 & 0,92 \\
Eq. 7 & 6,27 & $-2,93$ & 0,80 & 0,79 & 9,03 & $-0,27$ & 0,72 & 0,91 & 7,37 & $-0,83$ & 0,96 & 0,97 \\
\hline
\end{tabular}

\begin{tabular}{|c|c|c|c|c|c|c|c|c|c|c|c|c|}
\hline & \multicolumn{4}{|c|}{ Setembro/05 } & \multicolumn{4}{|c|}{ Outubro/05 } & \multicolumn{4}{|c|}{ Novembro/05 } \\
\hline Equação & MSE & MBE & $\mathbf{r}^{2}$ & d & RMSE & MBE & $\mathbf{r}^{2}$ & d & RMSE & MBE & $\mathbf{r}^{2}$ & d \\
\hline Eq. 1 & 32,28 & $-30,59$ & 0,83 & 0,68 & & $-27,79$ & 0,11 & 0,24 & 7,80 & & 0,05 & 0,25 \\
\hline Eq. 2 & & $-19,89$ & 0,54 & 0,71 & & $-33,34$ & 0,00 & & & & 0,15 & 0,20 \\
\hline Eq. 3 & 34 & $-9,79$ & 0,55 & 0,81 & 57 & $-22,71$ & 0,00 & 0,2 & & & 0,15 & 0,24 \\
\hline & & & 0,80 & & & $-15,84$ & 0,06 & & & & 0,06 & 0,32 \\
\hline & & -7 & 0,70 & 0,8 & & -16 & 0,01 & & & & 0,11 & 0,30 \\
\hline & & 0,1 & 0,86 & 0,9 & & & 0,31 & & & & 0,01 & 0,42 \\
\hline \multirow[t]{2}{*}{ Eq. 7} & & $-16,01$ & 0,79 & & & $-17,99$ & 0,05 & & & & 0,07 & 0,31 \\
\hline & \multicolumn{4}{|c|}{ Janeiro/06 } & \multicolumn{4}{|c|}{ Fevereiro/06 } & \multicolumn{4}{|c|}{ Março/06 } \\
\hline & & MBE & $\mathbf{r}^{2}$ & d & & MBE & $\mathbf{r}^{2}$ & d & & & $\mathbf{r}^{2}$ & d \\
\hline Eq. 1 & & $-34,48$ & 0,18 & 0,17 & & $-38,34$ & 0,15 & 0,29 & & & 0,16 & 0,27 \\
\hline & & $-45,82$ & 0,04 & & & $-51,95$ & 0,01 & 0,2 & & & 0,29 & \\
\hline & & $-35,80$ & 0,04 & & & $-41,95$ & 0,01 & & & & 0,29 & \\
\hline Eq. 4 & 23,83 & $-23,07$ & 0,15 & 0,23 & 28,87 & $-27,44$ & 0,15 & 0,36 & 27,91 & $-25,14$ & 0,18 & 0,32 \\
\hline & 26,20 & $-25,57$ & 0,08 & 0,22 & 32,23 & $-30,92$ & 0,00 & 0,33 & 31,05 & $-28,53$ & 0,24 & 0,30 \\
\hline Eq. 6 & & & 0,25 & 0,15 & & & 0,03 & 0,41 & & 1,22 & 0,10 & 0,20 \\
\hline Eq. 7 & 26,06 & $-25,38$ & 0,14 & 0,22 & 31,25 & $-29,93$ & 0,00 & 0,34 & 30,17 & $-27,64$ & 0,19 & 0,31 \\
\hline
\end{tabular}

\begin{tabular}{lcccccccc}
\multicolumn{1}{c}{ Abril/06 } & \multicolumn{1}{c}{ Maio/06 } \\
\hline Equação & RMSE & MBE & $\mathbf{r}^{2}$ & d & RMSE & MBE & $\mathbf{r}^{2}$ & d \\
Eq. 1 & 33,48 & $-31,25$ & 0,02 & 0,35 & 23,11 & $-19,74$ & 0,92 & 0,81 \\
Eq. 2 & 45,09 & $-42,53$ & 0,61 & 0,27 & 26,92 & $-17,84$ & 0,79 & 0,69 \\
Eq. 3 & 35,86 & $-32,46$ & 0,61 & 0,30 & 21,50 & $-8,64$ & 0,79 & 0,73 \\
Eq. 4 & 23,44 & $-19,90$ & 0,09 & 0,41 & 14,42 & $-5,25$ & 0,92 & 0,90 \\
Eq. 5 & 26,12 & $-22,44$ & 0,41 & 0,37 & 18,09 & $-0,30$ & 0,88 & 0,81 \\
Eq. 6 & 12,09 & 5,44 & 0,07 & 0,45 & 17,14 & 13,56 & 0,93 & 0,89 \\
Eq. 7 & 25,52 & $-22,25$ & 0,12 & 0,39 & 15,63 & $-6,12$ & 0,91 & 0,88 \\
\hline
\end{tabular}


Tabela 4 - Estatísticas relacionando os valores da radiação de onda longa atmosférica observados e estimados pelas equações de Brunt (1932) (Equação 1), Swinbank (1963) (Equação 2), Idso e Jackson (1969) (Equação 3), Brutsaert (1975) (Equação 4), Satterlund (1979) (Equação 5), Idso (1981) (Equação 6) e Prata (1996) (Equação 7), para os meses de junho de 2005 a maio de 2006 no sítio de floresta. A raiz do erro médio quadrático (RMSE) e o erro de viés médio (MBE) estão em W m², o coeficiente de determinação (r2) e o índice de concordância (d) são adimensionais.

\begin{tabular}{lcccccccccccc}
\hline \multicolumn{4}{c}{ Junho/05 } & \multicolumn{4}{c}{ Julho/05 } & \multicolumn{4}{c}{ Agosto/05 } \\
\hline Equação & RMSE & MBE & $\mathbf{r}^{2}$ & d & RMSE & MBE & $\mathbf{r}^{2}$ & d & RMSE & MBE & $\mathbf{r}^{2}$ & d \\
Eq. 1 & 19,21 & $-18,91$ & 0,66 & 0,40 & 15,96 & $-14,40$ & 0,83 & 0,80 & 14,48 & $-13,14$ & 0,92 & 0,90 \\
Eq. 2 & 29,25 & $-28,94$ & 0,50 & 0,27 & 16,24 & $-12,93$ & 0,65 & 0,75 & 12,16 & $-7,98$ & 0,85 & 0,90 \\
Eq. 3 & 18,77 & $-18,26$ & 0,50 & 0,39 & 10,29 & $-2,80$ & 0,65 & 0,88 & 9,25 & 2,83 & 0,85 & 0,94 \\
Eq. 4 & 8,76 & $-8,06$ & 0,64 & 0,64 & 7,17 & $-0,59$ & 0,81 & 0,95 & 6,38 & 0,44 & 0,91 & 0,98 \\
Eq. 5 & 11,53 & $-10,91$ & 0,57 & 0,53 & 8,99 & 2,66 & 0,74 & 0,90 & 9,54 & 4,35 & 0,89 & 0,93 \\
Eq. 6 & 17,10 & 16,80 & 0,70 & 0,37 & 19,10 & 18,04 & 0,87 & 0,76 & 18,88 & 17,95 & 0,93 & 0,85 \\
Eq. 7 & 12,81 & $-12,29$ & 0,61 & 0,51 & 8,16 & $-4,65$ & 0,84 & 0,93 & 7,89 & $-5,06$ & 0,93 & 0,96 \\
\hline
\end{tabular}

\begin{tabular}{lcccccccccccc}
\multicolumn{4}{c}{ Setembro/05 } & \multicolumn{4}{c}{ Outubro/05 } & \multicolumn{3}{c}{ Dezembro/05 } \\
\hline Equação & RMSE & MBE & $\mathbf{r}^{2}$ & $\mathbf{d}$ & RMSE & MBE & $\mathbf{r}^{2}$ & d & RMSE & MBE & $\mathbf{r}^{2}$ & d \\
Eq. 1 & 25,99 & $-24,24$ & 0,79 & 0,71 & 30,56 & $-29,51$ & 0,11 & 0,17 & 35,77 & $-35,58$ & 0,71 & 0,19 \\
Eq. 2 & 22,81 & $-19,26$ & 0,58 & 0,71 & 35,48 & $-33,38$ & 0,15 & 0,14 & 47,90 & $-47,64$ & 0,69 & 0,14 \\
Eq. 3 & 14,97 & $-8,33$ & 0,58 & 0,84 & 25,53 & $-22,24$ & 0,15 & 0,17 & 37,34 & $-37,00$ & 0,69 & 0,17 \\
Eq. 4 & 14,20 & $-10,76$ & 0,77 & 0,86 & 19,63 & $-17,88$ & 0,12 & 0,23 & 25,47 & $-25,15$ & 0,65 & 0,24 \\
Eq. 5 & 12,59 & $-7,18$ & 0,69 & 0,87 & 20,80 & $-18,71$ & 0,14 & 0,21 & 29,18 & $-28,83$ & 0,16 & 0,21 \\
Eq. 6 & 10,90 & 6,75 & 0,83 & 0,92 & 7,50 & 3,72 & 0,06 & 0,30 & 3,31 & 0,96 & 0,79 & 0,73 \\
Eq. 7 & 17,48 & $-15,35$ & 0,80 & 0,81 & 23,47 & $-22,26$ & 0,09 & 0,21 & 29,00 & $-28,76$ & 0,89 & 0,22
\end{tabular}

\begin{tabular}{lcccccccccccc}
\multicolumn{1}{c}{ Janeiro/06 } & \multicolumn{4}{c}{ Fevereiro/06 } & \multicolumn{4}{c}{ Março/06 } \\
\hline Equação & RMSE & MBE & $\mathbf{r}^{2}$ & $\mathbf{d}$ & RMSE & MBE & $\mathbf{r}^{2}$ & $\mathbf{d}$ & RMSE & MBE & $\mathbf{r}^{2}$ & $\mathbf{d}$ \\
Eq. 1 & 38,63 & $-38,13$ & 0,02 & 0,16 & 36,78 & $-36,40$ & 0,01 & 0,16 & 33,48 & $-32,91$ & 0,01 & 0,18 \\
Eq. 2 & 52,11 & $-51,66$ & 0,05 & 0,12 & 52,16 & $-51,77$ & 0,06 & 0,11 & 48,23 & $-47,79$ & 0,01 & 0,13 \\
Eq. 3 & 41,84 & $-41,26$ & 0,05 & 0,15 & 41,84 & $-41,33$ & 0,06 & 0,14 & 37,82 & $-37,24$ & 0,01 & 0,16 \\
Eq. 4 & 28,42 & $-27,76$ & 0,03 & 0,20 & 27,06 & $-26,54$ & 0,01 & 0,20 & 23,84 & $-23,09$ & 0,00 & 0,24 \\
Eq. 5 & 32,34 & $-31,77$ & 0,04 & 0,18 & 31,89 & $-31,41$ & 0,04 & 0,17 & 28,51 & $-27,90$ & 0,00 & 0,21 \\
Eq. 6 & 5,99 & $-0,83$ & 0,01 & 0,33 & 5,06 & 1,65 & 0,00 & 0,32 & 7,64 & 4,84 & 0,02 & 0,37 \\
Eq. 7 & 31,17 & $-30,63$ & 0,01 & 0,19 & 29,69 & $-29,23$ & 0,01 & 0,18 & 20,22 & $-19,43$ & 0,03 & 0,28 \\
\hline
\end{tabular}

\begin{tabular}{lcccccccc}
\multicolumn{1}{c}{ Abril/06 } & \multicolumn{4}{c}{ Maio/06 } \\
\hline Equação & RMSE & MBE & $\mathbf{r}^{\mathbf{2}}$ & $\mathbf{d}$ & $\mathbf{R M S E}$ & $\mathbf{M B E}$ & $\mathbf{r}^{\mathbf{2}}$ & $\mathbf{d}$ \\
Eq. 1 & 29,88 & $-29,28$ & 0,00 & 0,22 & 20,00 & $-18,70$ & 0,94 & 0,82 \\
Eq. 2 & 45,79 & $-45,17$ & 0,16 & 0,15 & 27,27 & $-23,67$ & 0,86 & 0,68 \\
Eq. 3 & 35,41 & $-34,56$ & 0,16 & 0,18 & 19,09 & $-13,97$ & 0,86 & 0,77 \\
Eq. 4 & 20,71 & $-19,79$ & 0,00 & 0,28 & 10,39 & $-5,81$ & 0,94 & 0,93 \\
Eq. 5 & 25,96 & $-25,12$ & 0,07 & 0,23 & 13,39 & $-4,87$ & 0,91 & 0,86 \\
Eq. 6 & 10,26 & 8,87 & 0,10 & 0,45 & 17,18 & 16,15 & 0,95 & 0,87 \\
Eq. 7 & 23,05 & $-22,29$ & 0,00 & 0,26 & 12,25 & $-8,24$ & 0,94 & 0,91 \\
\hline
\end{tabular}

dados utilizados na avaliação das equações, comprometendo o desempenho das mesmas. As equações que levam em consideração a pressão de vapor d'água e a temperatura do ar tiveram melhor desempenho do que as que utilizam apenas a temperatura do ar, como é o caso das equações de Swinbank (1963) e Idso e Jackson (1969). As equações de Brutsaert (1975), Idso (1981) e Prata (1996) foram as que apresentaram melhor desempenho, obtendo os maiores índices de concordância, e sendo, portanto, as equações mais indicadas para a estimativa da radiação de onda longa atmosférica no sudoeste da Amazônia.

O baixo desempenho das equações avaliadas na estação chuvosa deixa claro a pouca utilidade dessas equações, que foram desenvolvidas para condições de céu claro, para estimar a radiação de onda longa atmosférica na região Amazônica, onde predominam as condições de céu nublado. Por sua vez, torna-se evidente também, a necessidade de novas parametrizações para estimar a radiação de onda longa atmosférica que possam ser utilizadas para todas as condições de cobertura do céu.

\section{AGRADECIMENTOS}

Agradecemos ao Programa LBA pela disponibilização dos dados e aos estudantes bolsistas de iniciação científica do Programa LBA de Rondônia pela coleta dos dados, sem 


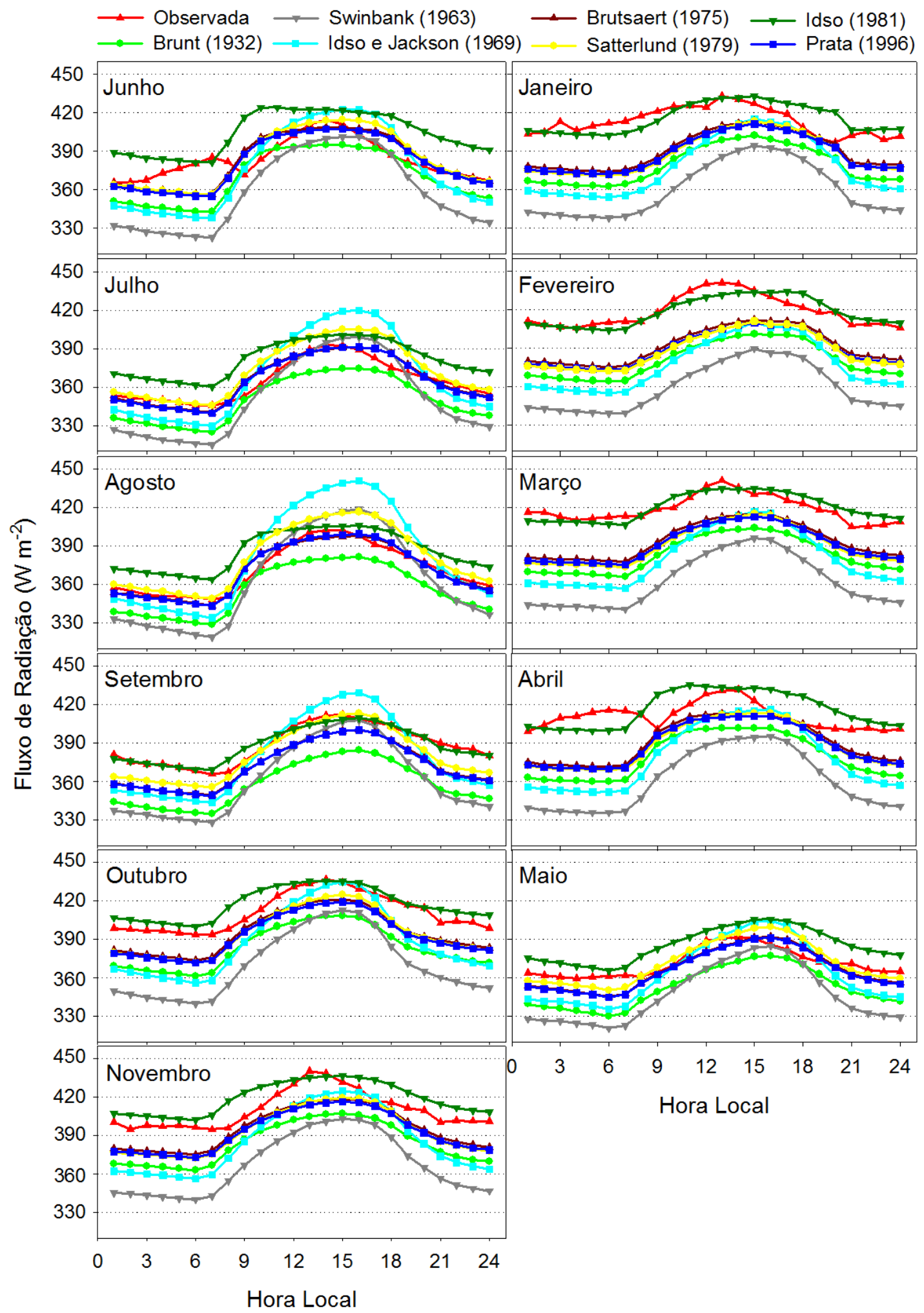

Figura 4 - Ciclo médio diário da radiação de onda longa atmosférica observada e estimada pelas equações avaliadas para os meses de junho de 2005 a maio de 2006 no sítio de pastagem. 


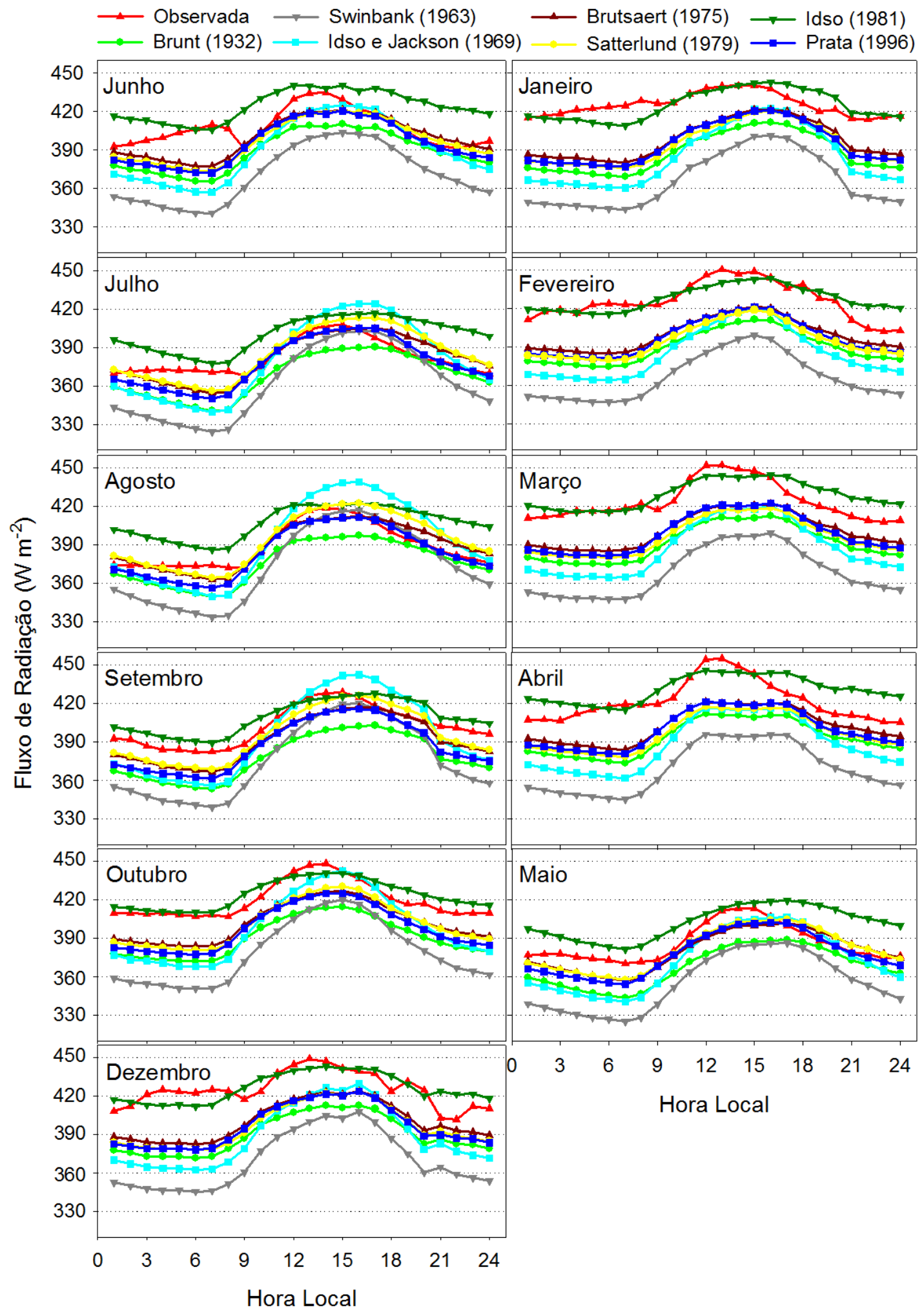

Figura 5 - Ciclo médio diário da radiação de onda longa atmosférica observada e estimada pelas equações avaliadas para os meses de junho de 2005 a maio de 2006 no sítio de floresta. 
os quais essa pesquisa não poderia ser realizada. O primeiro autor agradece ao CNPq pelo apoio financeiro dado através da concessão de bolsa de mestrado.

\section{REFERÊNCIAS BIBLIOGRÁFICAS}

ANDRÉ, R. G. B. et al. Balanço de radiação sobre a floresta Amazônica (estações seca e úmida). Revista Brasileira de Meteorologia, v. 3, n. 2, p. 269-274, 1988.

BASTABLE, H. G. et al. Observations of climate albedo, and surface radiation over cleared and undisturbed Amazonian forest. International Journal of Climatology, v. 13, p. 783-796, 1993.

BRUNT, D. Notes on radiationin the atmosphere. Quarterly Journal Resource Meteorological Society, v. 58, p. 389-418, 1932.

BRUTSAERT, W. On a derivable formula for long-wave radiation from clear skies. Water Resources Research, v. 11, n. 5, p. 742-744, Oct. 1975.

CORREIA, F. W. S. Estudo do Balanço de Radiação em Área de Policultivo na Amazônia. 2000. 137 f. Dissertação (Mestrado em Meteorologia), Instituto Nacional de Pesquisas Espaciais (INPE), São José dos Campos, 2000.

CULF A. D.; FISCH, G.; HODNETT, M. H. The albedo of Amazonian forest and ranchland. Journal of Climate, v. 6, n. 8, p. 1544-1554, May. 1995.

CULF, A. D. et al. Radiation, temperature and humidity over forest and pasture in Amazonia. In: Amazonian deforestation and climate (eds. J.H.C. Gash, C. A. Nobre, J. M. Roberts, R. L. Victoria). Chichester: John Wiley, p. 175-191, 1996.

CULF, A. D.; FISCH, G.; MALHI, Y.; NOBRE, C. A. The influence of the atmospheric boundary layer on carbon dioxide concentrations over a tropical forest. Agricultural and Forest Meteorology, v. 85, p. 149-158, 1997.

FEITOSA, J. R. P. Balanço de energia e evapotranspiração em áreas de pastagem e de floresta densa na Amazônia central. 1996. 95 f. Dissertação (Mestrado em Meteorologia), Faculdade de Meteorologia, Universidade Federal da Paraíba, Campina Grande, 1996.

GALVÃO, J. A. C.; FISCH, G. Balanço de radiação em área de pastagem na Amazônia. Revista Brasileira de Agrometeorologia, v. 8, n. 1, p. 1-10, 2000.

IDSO, S. B.; JACKSON R. D. Thermal radiation from the atmosphere. Journal Geophysics Research, v. 74, n. 23, p. 5397-5403, 1969.
IDSO, S. B. A set of equations for full spectrum and 8 to $14 \mu \mathrm{m}$ and 10.5 to $12.5 \mu \mathrm{m}$ thermal radiation from cloudless skies. Water Resources Research, v. 17, n. 2, p. 295-304, 1981.

IQBAL, M, An Introduction to Solar Radiation. Toronto: Academic Press, 416 f, 1983.

MANZI, A. O. et al. Um estudo sobre o balanço de radiação da floresta Amazônica. São José dos Campos. (INPE-3956PRE/974). 1986.

PRATA, A .J. A new long-wave formula for estimating downward clear-sky radiation at the surface. Quarterly Journal Resource Meteorological Society, v. 122, p. 1127-1151, 1996.

RESCHKE, G. A. Influência do desmatamento no balanço de radiação e nos fluxos de calor sensível e calor latente em Ji-Paraná, Rondônia. 1996. 51 f. Dissertação (Mestrado em Meteorologia Agrícola), Faculdade de Agronomia, Universidade Federal de Viçosa, Viçosa, 1996.

RIBEIRO, J. B. M. Análise comparativa das características microclimáticas entre áreas de floresta e de pastagem na Amazônia. 1994. 67 f. Dissertação (Mestrado em Meteorologia Agrícola), Faculdade de Agronomia, Universidade Federal de Viçosa, Viçosa, 1994.

SATTERLUND, D. R. An improved equation for estimating long-wave radiation from the atmosphere. Water Resources Research, v. 15, p. 1649-1650, 1979.

SILVA DIAS, M.A.F.; COHEN, J. C. P.; GANDU, A. W. Interações entre Nuvens, Chuvas e a Biosfera na Amazônia. Acta Amazonica. v. 35, n. 2, p. 215-222, 2005.

SWINBANK, W. C. Long-Wave Radiation from clear skies. Quarterly Journal Resource Meteorological Society, v. 89, n. 381, p. 339-348, July. 1963.

TETENS, O. Uber cinige meterorologische Begriffe. Z. Geophys., n.6, p. 297-309, 1930.

VON RANDOW, C. et al. Comparative measurements and seasonal variations in energy and carbon exchange over forest and pasture in South West Amazonia. Theoretical and Applied Climatology. p. 1-22, 2004.

VON RANDOW, R. C. S.; ALVALÁ, R. C. S. Estimativa da radiação de onda longa atmosférica no Pantanal Sul Mato-Grossense durante os períodos secos de 1999 e 2000. Revista Brasileira de Meteorologia, v. 21, n. 3b, p. 398-412, 2006. 